\title{
The HNF-3 Gene Family of Transcription Factors in Mice: Gene Structure, cDNA Sequence, and mRNA Distribution
}

\author{
Klaus H. Kaestner, Holger Hiemisch, Bruno Luckow, and Günther Schütz ${ }^{2}$ \\ Division of Molecular Biology of the Cell I, German Cancer Research Center, Im Neuenheimer Feld 280, D-69120 Heidelberg, Germany
}

Received October 26, 1993; revised January 1, 1994

The rat HNF-3 (hepatocyte nuclear factor 3) gene family encodes three transcription factors known to be important in the regulation of gene expression in liver and lung. We have cloned and characterized the mouse genes and CDNAs for HNF-3 $\alpha, \beta$, and $\gamma$ and analyzed their expression patterns in various adult tissues and mouse embryonic stages. The HNF-3 proteins are highly conserved between mouse and rat, with the exception of the amino terminus of HNF-3 $\gamma$, which in mouse is more similar to those of HNF-3 $\alpha$ and $\beta$ than to the amino termini of the rat HNF-3 $\gamma$ protein. The mouse HNF-3 genes are small and contain only two or three $(\mathrm{HNF}-3 \beta)$ exons with conserved intron-exon boundaries. The proximal promoter of the mouse HNF$3 \beta$ gene is remarkably similar to that of the previously cloned rat HNF-3 $\beta$ gene, but is different from the promoters of the HNF-3 $\alpha$ and $\gamma$ genes. The mRNA distribution of the mouse HNF-3 genes was analyzed by quantitative RNase protection with gene-specific probes. While HNF $-3 \alpha$ and $\beta$ are restricted mainly to endoderm-derived tissues (lung, liver, stomach, and small intestine), HNF-3 $\gamma$ is more extensively expressed, being present additionally in ovary, testis, heart, and adipose tissue, but missing from lung. Transcripts for HNF $-3 \beta$ and $\alpha$ are detected most abundantly in midgestation embryos (Day 9.5), while HNF-3 $\gamma$ expression peaks around Day 15.5 of gestation. (c) 1994 Academic Press, Inc.

\section{INTRODUCTION}

Much progress has been made toward understanding liver-specific gene expression through biochemical analysis of the promoters and enhancers of genes such as those encoding albumin or transthyretin and the subse-

The nucletide sequences reported in this paper have been deposited in the EMBL Data Base under Accession Nos. X74936, X74937, X74938, X76684, X76685, and X76686.

${ }^{1}$ Present address: Medizinische Poliklinik, Abt. Klinische Biochemie, Schillerstrasse 42, D-80336 Munich, Germany.

${ }^{2}$ To whom correspondence should be addressed. Telephone: ++49 6221-423411. Fax: ++49-6221-423404. quent isolation of the transcription factors important in their regulation (for review see Lai and Darnell, 1991; Sladek and Darnell, 1992; De Simone and Cortese, 1991).

The major liver-enriched transcription factors identified so far include HNF-1, a POU-homeodomain protein; C/EBP $\alpha$ and $\beta$, which are bZip proteins; HNF-4, a member of the thyroid-steroid hormone receptor superfamily; and HNF-3, a representative of the HNF-3/forkhead class of DNA-binding proteins (Lai and Darnell, 1991; Sladek and Darnell, 1992; De Simone and Cortese, 1991, and references therein). The HNF-3 proteins were first identified by their ability to bind to important promoter elements in the $\alpha_{1}$-antitrypsin and transthyretin genes (Costa et al., 1989). Other target sites for HNF-3 have been described in the $\alpha$-fetoprotein, albumin, tyrosine aminotransferase, phosphoenolpyruvate kinase, transferrin, and HNF- $1 \alpha$ and HNF- $3 \beta$ genes (Costa et al., 1989; Herbst et al. 1991; Nitsch et al., 1993; Ip et al., 1990; Auge-Gouillou et al., 1993; Kuo et al., 1992; Pani et al., 1992b) as well as the lung-specific gene CC10 (Sawaya et al., 1993). From comparison of the observed HNF-3 binding sites, a consensus element, TATTGA ${ }^{\mathrm{C}}$ / ${ }_{\mathrm{T}} \mathrm{TT}^{\mathrm{A}} /{ }_{\mathrm{T}} \mathrm{G}$, has been derived (Costa et al., 1989), although deviations from this consensus sequence have been identified (Nitsch et al., 1993; Pani et al., 1992b). The HNF-3 DNA-binding activities were purified from rat liver extracts and the rat cDNAs subsequently cloned and sequenced (Lai et al., 1990, 1991). The three binding activities observed in gel retardation assays were found to correspond to three proteins encoded by independent genes, which were termed HNF- $3 \alpha, \beta$, and $\gamma$ (Lai et al., 1991). Deletion analysis of HNF- $3 \alpha$ demonstrated that the region between amino acids 124 and 288 is essential for DNA binding (Lai et al., 1990). Subsequent sequence comparison revealed striking similarities between HNF3 and the Drosophila melanogaster gene forkhead within the DNA binding domain (Lai et al., 1991; Weigel and Jäckle, 1990), for which 100 of 110 amino acids are identical between HNF-3 $\beta$ and forkhead. The Drosophila gene forkhead has been described as a region-specific homeotic gene and is required for the proper formation of the terminal structures of the Drosophila embryo 
A 1 CGCCGCGCCGCGCCGCCGCCGCCGCGCACGCCGCGCCCCGCAGCGCCGGGCTTCCTCCTCGCCCCGGGTGGCGCTGGGCCCTCGAGCGCTCCGGTTACCGCAGCGGCTCCGCGCCCCTCCC 121 CCGCCCCGAGCAGCGCACCCGCCCGTCGCTCCGCACAGGGTTGGATGGTTGTGTCGGCCGGGCTGGCTCCAGGATGTTAGGGACTGTGAAGATGGAGGGGCATGAGAGCAACGACTGGAA

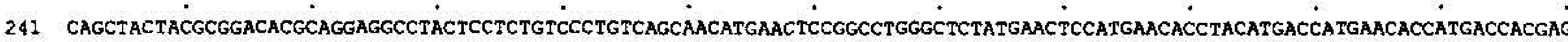

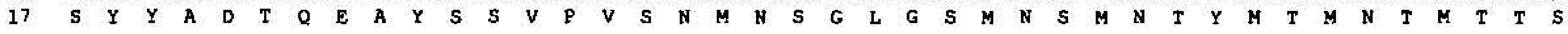

361 CGGCAACATGACCCCGGCTTCCTTCAACAT́TTCCTACGCCAACACGGCTITAGGGCCGGCCTGAGTCCCGGTGCTGTGGCTGGCATGCCAGGGGCCTCTGCAGGCGCCATGAACAGCAT

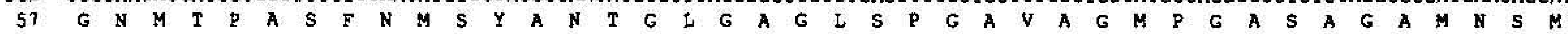

481 GACTGCGGCGGGCGTCACGGCCATGGGTACGGCGCTGAGCCCGGGAGGCÁTGGGCTCCATGGGCGCGCAGCCCGTCACCTCCATGAACGGCCTGGGTCCCTACGCCGCCGCCATGAACCC

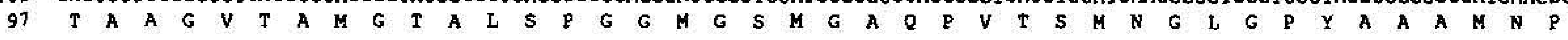

601 GTGCATGAGTCCCATGGCGTACGCGCCGTCCAACCTGGGCCGCAGCCGCGCGGgGgGCGGCGGCGACGCCAAGACATTCAAGCGCAGCTACCCTCACGCCAAGCCGCCTTACTCCTACAT

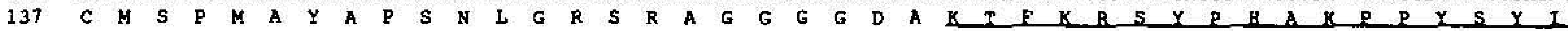

721 CrCGCTCATCACGATGGCCATCCAGCAGGCGCCCAGCAAGATGCTCACGĆTGAGCGAGATCTACCAGTGGATCATGGACCTCTICCCCTATTACCGCCAGACCAGCAGCGCTGGCAGAA

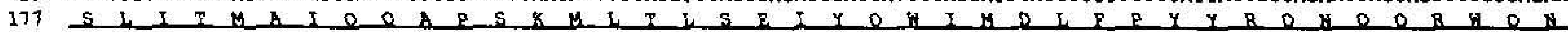

643 CTCCATCCGCCACTCGCTGTCCTTCAACGATTGTTTCGTCAAGGTGGCACGATCCCCAGACAAGCCAGGCAAGGGCTCCTACTGGACGCTGCACCCGGACTCCGGCAACATGTTCGAGAA

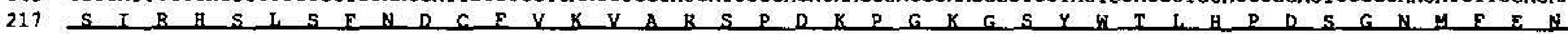

961 CGGCTGCTACTTGCGCCGCCAAAAGCGCTTCAAGTGTGAGAAGCAGCCGGGGGCCGGAGGTGGGAGTGGGGGCGGGGCTCCAAAGGGGGCCCAGAAAGTCGCAAGGACCCCTCAGGCCC

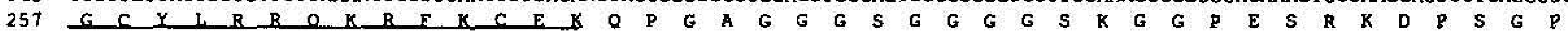

1081 GGGGAACCCCAGCGCCGAGTCACCCCTTCATTGGGGTGTGCACGGAAAGGCTAGCCAGCTAGAGGSCGCGCCGGCCCCCGGGGCCCGCCGCCAGCCCCCAGACTCTGGACCACAGCGGGGC

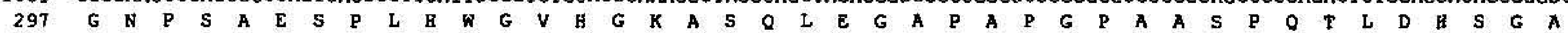

1201 CACGGCGACAGGGGGCGCTTCGGAGTTGAAGTCTCCAGCGTCTTCATCTGCGCCCCCCATAAGCTCCGGGCCAGGGGCCTGGCATCTGTACCCCCCTCTCACCCGGCTCACGGCCTGGC

337 I A

1321 ACCCCACGAATCTCAGCTGCATCTGAAAGGGATCCCCACTACTCCTTTAATCACCCCTTCTCCATCAACAACCTCATGTCCTCCTCCGAGCAACAGCACAAGCTGGACTTCAAGGCATA

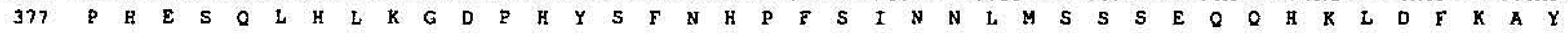

1441 CGAGCAGGCGCTGCAGTACTCTCCTTATGGCGCTACCTTGCCCGCCAGTCTGCCCCTTGGCAGCGCCTCAGTGGCCACGAGGAGCCCCATCGAGCCCTCAGCCCTGGAGC̈CAGCCTACTA

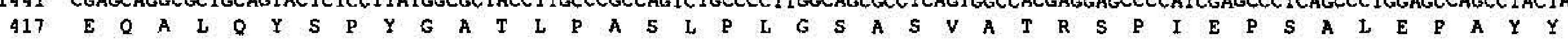

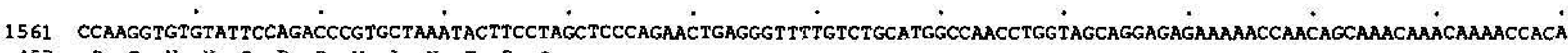

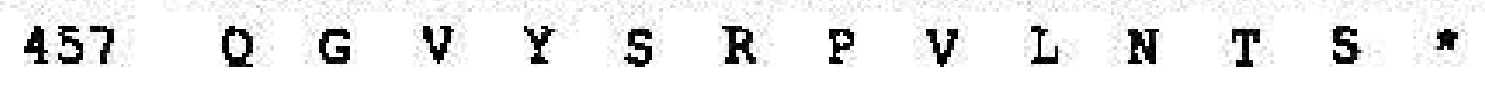

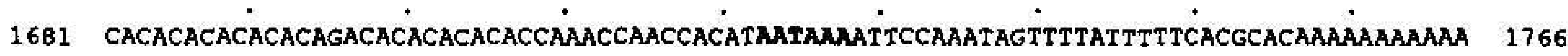

B I TTGACGACCAGGGCGGCCAGACCACGCGAGTCCTACGCGCCTCCTTGAGGCCGCCCCGGGACTTAACTGTAACGGGGAGGGGCCTCCGGAGCAGCGGCCAGCGAGTTAAAGTATGCTGGGä

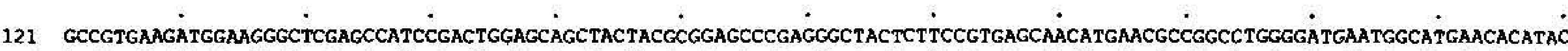

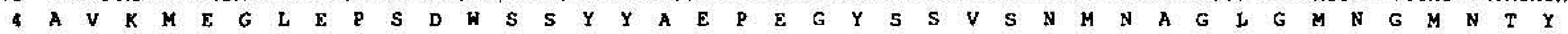

241 ATGAGCATGTCCGCGGCTGCCATGGGCGGCGGTTCCGGCAACATGAGCGCGGGCTCCATGAACATGTCATCCTATGTGGGCGCTGGAATGAGCCCGTCGCTAGCTGGCATGTCCCCGGGC

44 M S M S A A A M G G G S G N M S A G S M N M S S Y V G A G M S $P$ P $S$ L A G M $S$ P $G$

361 GCCGGCGCCATGGCGGGCATGAGCGGCTCAGCCGGGGCGGCCGGCGTGGCGGGCATGGGACCTCACCTGAGTCCGAGTCTGAGCCCGCTCGGGGGACAGGCGGCCGGGGCCATGGGTGGC

84 A G A M A G M S G S A G A A G V A G M G P

481 CTTGCCCCCTACGCCAACATGAaCTCGATGAGCCCCATGTACGGGCAGGCGGGCCTGAGCCGCGCTCGGGACCCCAAGACATACCGACGCAGCTACACACACGCCAAACCTCCCTACTCG

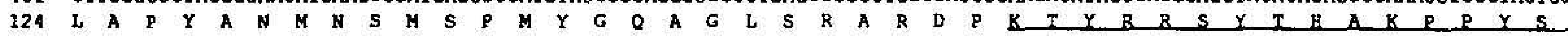

601 TACATCTCGCTCATCACCATGGCCATCCRGCAGAGCCCCAACAAGATGCTGACGCTGAGCGAGATCTATCAGTGGATCATGGACCTCTTCCCTTTCTACCGGCAGAACCAGCAGCECTGG

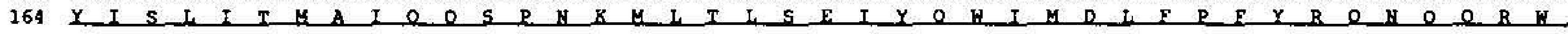

721 CAGAACTCCATCCGCCACTCTCTCTCCTTCAACGACTGCTTTCTCAAGGTGCCCCGCTCGCCAGACAAGCCTGGCAAGGGCTCCTTCTGGACCCTGCACCCAGACTCGGGCAACATGTTC

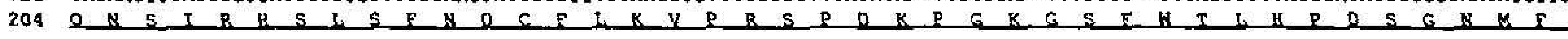

841 GAGAACGGCTGCTACCTGCGCCGCCAGAAGCGCTTCAAGTGTGAGAAGCAACTGGCACTGAAGGAAGCCGCGGGTGCGGCCAGTAGCGGAGGCAAGAAGACCGCTCCTGGGTCCCAGGCC

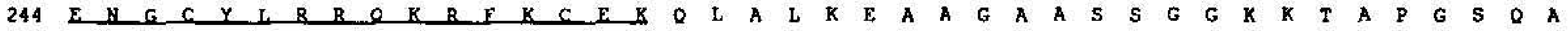

961 TCTCAGGCTCAGCTCGGGGAGGCCGCGGGCTCGGCCTCCGAGACTCCGGCGGGCACCGAGTTCCCCCCATTCCAGCGCTTCTCCGTGTCAGGAGCACAAGCGAGGTGGCCTAAGCGAGCTA 284 S Q A Q L G E A A G S A S E T P A G T E S P H S S A S P C Q E H K R G G L S E L

1081 AAGGGAGCACCTGCCTCTGCGCTGAGTCCTCCCGAGCCGGCGCCCTCGCCTGGGCAGCAGCAGCAGGCTGCAGCCCACCTGCTGGGCCCACCTCACCACCCAGGCCTGCCACCAGAGGCC

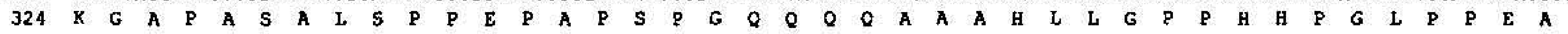

1201 CACCTGAAGCCCGAGCACCATTACGCCTTCAACCACCCCTTCTCTATCAACAACCTCATGTCGTCCGAGCAGCAACATCACCACAGCCACCACCACCATCAGCCCCACAMAATGGACCTC

364 H L

1321 AAGGCCTACGAACAGGTCATGCACTACCCAGGGGGCTATGGTTCCCCCATGCCAGGCAGCTTGGCCATGGGCCCAGTCACGAACAAAGCGGGCCTGGATGCCTCGCCCCTGGCTGCAGAC

404 K A Y E E Q V M A $Y$ Y P G G Y G S P M P G S

1441 ACTTCCTACTACCAAGGAGTGTACTCCAGGCCTATTATGAACTCATCCTAAGAAGATGGCTTTCAGGCCCTGCTAGCTCTGGTCACTGGGGACAAGGGAAATGAGAGGCTGAGTGGAGAC

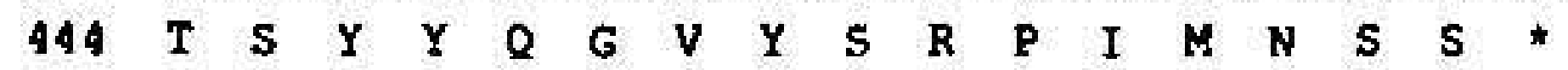

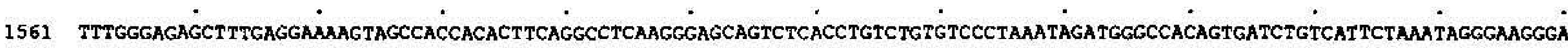

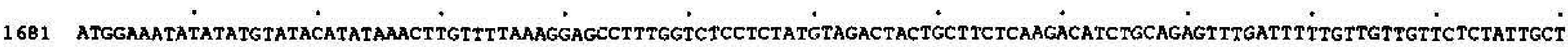

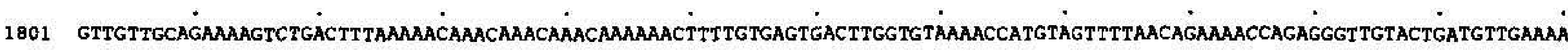

1921 GaGgAaAGAAAAATAATGTAAGAGTCTGGTGTACCGGACCAGGAGAAAGGaGAAAAACACATCCCATTCTGGACATGGTGAAATCCAGGTCTCGGGTCTGATTTAATTTATGGTTTCTGC 


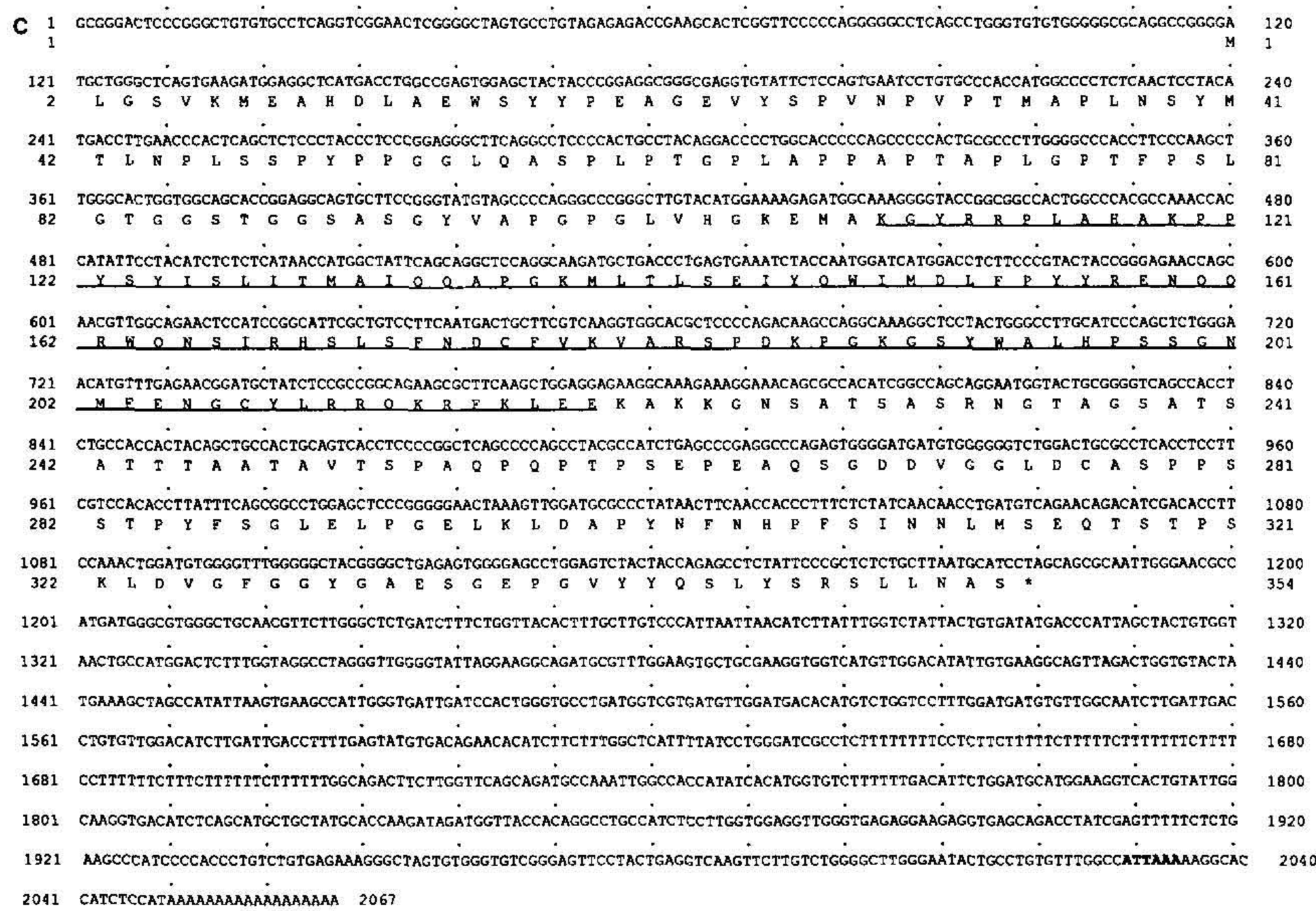

FIG. 1. Nucleotide and translated amino acid sequences of the mouse HNF-3 $\alpha, \beta$, and $\gamma$ cDNAs. Shown is the sequence of the sense strand

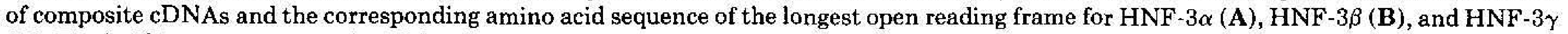
(C). The boldface sequences in $\mathbf{A}$ and $\mathbf{C}$ indicate potential polyadenylation signals. The sequences of the 110-amino-acid forkhead DNA binding domains are underlined.

(Jürgens and Weigel, 1988; Weigel et al., 1989). The forkhead gene is expressed in ectodermal as well as endodermal portions of the gut, the yolk nuclei, the salivary glands, and certain cells of the nervous system. Subsequently, sequences closely related to the HNF-3/ forkhead DNA binding domain have been found in species ranging from yeast to man (Oliver et al., 1992; Knöchel et al., 1992; Dirksen and Jamrich, 1992; Ruiz i Altaba and Jessell, 1992; Häcker et al., 1992: Tao and Lai, 1992; Li et al., 1991, 1992; Li and Vogt, 1993; Kaestner et al., 1993; Clevidence et al., 1993). In mice, six HNF-3/forkhead homologues that have at least 57\% amino acid identity within the DNA binding domain with forkhead have been described (Kaestner et al., 1993).

The rat HNF-3 genes have been shown to be expressed, in addition to liver, in stomach, intestine, and lung (Lai et al., 1990, 1991), which are all tissues derived, at least in part, from embryonic endoderm. This fact, combined with the high degree of similarity to the $\mathrm{Dro}$ sophila gene forkhead, has led to the proposal that the HNF-3 genes are important in early endoderm and liver development in addition to their role in adult liver transcription (Lai and Darnell, 1991). This notion is supported by recent in situ hybridization studies on early postimplantation and midgestation mouse embryos (Sasaki and Hogan, 1993; Monaghan et al., 1993). The HNF-3 family members were found to be sequentially transcribed in the developing definite endoderm, HNF$3 \beta$ being the first gene to be activated, followed by HNF$3 \alpha$ and finally $\gamma$. Interestingly, HNF $-3 \beta$ and $\alpha$ mRNAs were also found in cells of the notochord and ventral neural epithelium, suggesting additional functions for these genes in mesoderm and neural axis formation (Monaghan et al., 1993). Recent experiments suggest that the very early expression of the HNF-3 proteins in the liver primordium is responsible for the reorganization of the chromatin structure, as the HNF-3 proteins are involved in the precise positioning of nucleosomes over the albumin enhancer only in those tissues where the enhancer is active (McPherson et al., 1993). As a prerequisite to further our understanding of the importance of the HNF-3 gene family in mouse development, through promoter analysis in transgenic mice and generation of null alleles via homologous recombination in embryonic stem cells, we have cloned and characterized cDNAs and genomic fragments encoding the mouse HNF-3 genes. In addition, we have quantitatively analyzed their expression pattern in adult mouse tissues and whole mouse embryos from midgestation to term. 
mHNF-3 $\alpha$
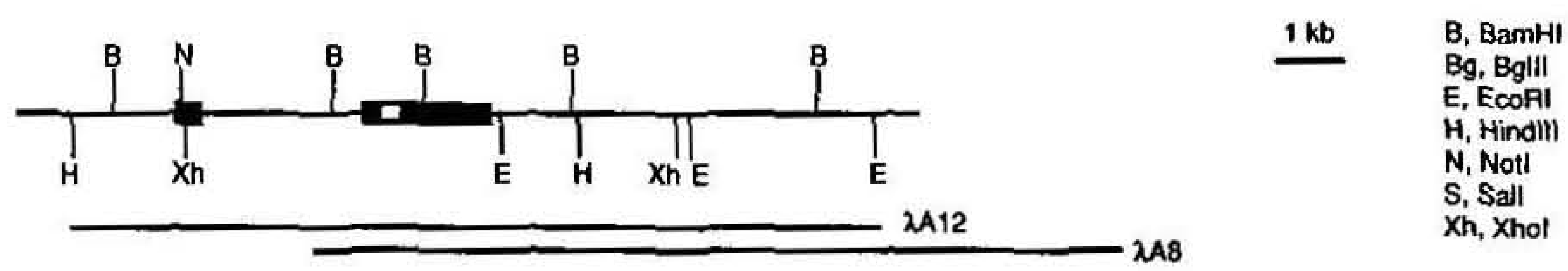

mHNF-3 $\beta$

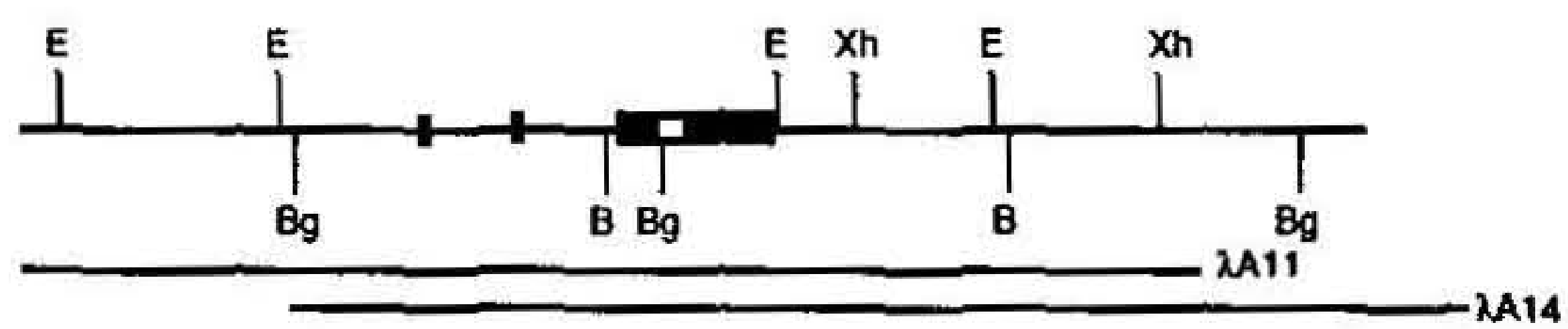

mHNF-3 $\gamma$

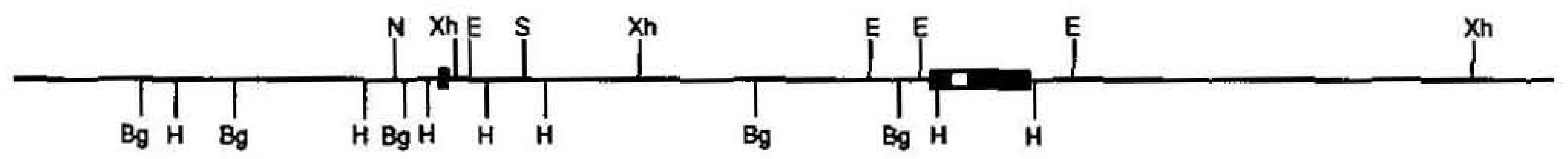

$\lambda E 8$

FIG. 2. Restriction map of the mouse HNF $-3 \alpha, \beta$, and $\gamma$ genes. The restriction map of each gene together with the extent of the lambda phage clones from which it was derived is shown. The exons of the three genes are shown as black boxes, and the forkhead DNA binding domains are indicated as white boxes.

\section{MATERIALS AND METHODS}

Library construction and cDNA and genomic cloning. A 300-nt probe corresponding to the forkhead domain of the mouse HNF- $3 \alpha$ gene originally obtained by PCR (Kaestner et al., 1993) was used to screen two mouse liver cDNA libraries (Ruppert et al., 1990; B. Luckow et al., 1994) using high-stringency hybridization and washing conditions (Church and Gilbert, 1984). Fourteen hybridizing lambda phages were purified and the cDNAs were subcloned into Bluescript. (Stratagene) and classified by dideoxy sequencing (Sanger et al., 1977). The complete coding as well as part of the untranslated regions of the mouse HNF- $3 \alpha, \beta$, and $\gamma$ cDNAs were sequenced after generating nested deletions of the cDNAs with exonuclease III (Henikoff, 1984).

Genomic clones containing the HNF- $3 \alpha$ and $\beta$ genes were obtained through screening of an amplified mouse 129/Ola library in $\lambda$ GEM-12

TABLE 1

Intron-Exon Boundaries of the HNF-3 Genes in Mice

HNF-3 $\alpha$

Exon 1 AACGCAGGAGgtgagaggcggg T Q $\mathrm{E}_{24}$ 2.5 -kb intron

Exon 2 24

HNF - $3 \beta$

Exon 1 GCGAGTTAAAgtt tectaaat .................... 1.5 -kb intron

Exon 2 $\mathrm{A}_{25} \mathrm{Y} S$

Exon 2 GGAGCCCGAGgtaagctcttgc E $\quad P \quad E_{23}$ $0.5-\mathrm{kb}$ intron $M_{1} L \quad G$

Exon 3 ctcggcttccagTATGCTGGGA GGCTACTCTT

HNF-3 $\gamma$

Exon 1

Exon 2 GGCGGGCGAGgtgtgtcctcgg 8-kb intron ctctccctctagGTGTATTCTC 
FIG. 3. Mapping of the transcription initiation sites of the mouse HNF-3 $\alpha, \beta$, and $\gamma$ genes. Synthetic oligonucleotide primers complementary to the sense strand of the cDNAs were end-labeled with ${ }^{32} \mathrm{P}$ to a specific activity of $10^{8} \mathrm{cpm} / \mu \mathrm{g}$. Primer extension analysis was carried out with $5 \mu \mathrm{g}$ of poly $\left(\mathrm{A}^{+}\right)$RNA from stomach or $5 \mu \mathrm{g}$ tRNA. Marker lanes contain the dideoxy nucleotide sequencing reactions of the first exon of each gene using the same primers. Arrows indicate the major start sites of transcription.

(Promega) kindly provided by Anton Berns (Amsterdam) using the probe and hybridization conditions as used for the cDNA library screen. An additional genomic library was constructed from murine embryonic stem cells. Genomic DNA was isolated from the murine embryonic stem cell line E14TG2a (Hooper et al., 1987), partially digested with the restriction endonuclease $\$ a u 3 A I$, size fractionated (16 to $23 \mathrm{~kb}$ fragments), and ligated into $\lambda$ Dash II (Stratagene) according to Frischauf (1987). Two million primary phages of this library were screened with various portions of the mouse HNF- $3 \gamma$ cDNA to assemble the complete HNF-3 $\gamma$ contig. The genomic lambda phages were mapped, and exon-containing fragments subcloned and sequenced to obtain the intron-exon boundaries.

Primer extension analysis. Primer extension analysis was carried out as described previously (Kaestner et al., 1989) using synthetic oligonucleotides and $5 \mu \mathrm{g}$ tRNA or poly $\left(\mathrm{A}^{+}\right)$RNA purified from stomach using oligo(dT) Dynabeads (Dynal). The primers used were complementary to nucleotides 64 to 85 of the HNF- $3 \alpha$ cDNA, nucleotides 61 to 83 of the HNF-3 $\beta$ cDNA, and nucleotides 61 to 83 of the HNF-3 $\gamma$ cDNA (see Fig. 1). To map the endpoint of the primer-extended product, dideoxy sequencing (Sanger et al., 1977) of genomic subclones containing the first exon of each gene was performed using the corresponding oligonucleotide as a primer.

RNA isolation and RNase protection analysis. Total RNA from a variety of mouse tissues or whole mouse embryos was isolated by centrifugation through a $\mathrm{CsCl}$ cushion after homogenization in guanidinium thiocyanate (Chirgwin et al., 1979). The quality of the RNA preparations was controlled by ethidium bromide staining of the $18 \mathrm{~S}$ and $28 \mathrm{~S}$ rRNAs after electrophoretic separation of the RNA in denaturing agarose gels. RNase protection analysis was performed as described previously (Kaestner et al., 1989) using [ $\alpha-{ }^{32}$ P]UTP-labeled antisense RNA probes derived from Bluescript (Stratagene) subclones containing 191 bp (HNF-3 $\alpha$, position 1165 to 1355), 221 bp (HNF-3 $\beta$, position 1169 to 1389 ), or 338 bp (HNF-3 $\gamma$, position 993 to 1330 ) of the mouse HNF-3 cDNAs. The probes chosen were from regions outside the conserved domains of the HNF-3 family to avoid the possibil. ity of cross-hybridization. The antisense probes were hybridized against $50 \mu \mathrm{g}$ (adult tissues) or $20 \mu \mathrm{g}$ (embryonic samples) total RNA

A

ACGATCCTTG CGCTGTAGCT CTGATGCCAC CACCCAGGTC CTTCTCCCGC AGCACAGCTC TTTGGGTCCA GAGCCCTGGC CTGTCCTCCA -396

AGGCACCGCC TATTTTTCCT TTTCTTCTTT TTTTTCCTTT TCTTTTCTTT CTCTTTTTTT CCTTTTCCAA AGGGGGTCAC ACACACACCC -306 CCAAT-BOX GCCCTATTTT CCTCTTTCCC TAGCCGCGAG GCTTAAACCA ATTATACTGC TTTGTAAACA AAGTGAGGGC CAGGTTTGGG GGAGGGGATG -216 GPIGGAGGGG CGCGGGGGGC GCGCAGGCGC TGGCGCGGCG GGGCGGGAGG CGCGGCGGCT GGACTGGCGG GCGGCCGCCT CACAGGTGCA -126

CCTCGGGCTT TGTAGGTGCG AGCGTCTTTG TGCGGCGGAC AAATGGGGAG AGGACGAGGA GGTGGGCACT CCGGCGACGT AAGATCCACA -36 SP1 1 TCAGCTCAAC TGCACTCGCT TCGCACAGGC CGCCCGCTCA CTTCCCGCGG AGGCGCTGCC GGGCGCCGGC TCCGCGGCCG CCTCCTGTCC +56 CCGGCGCTGC CCCCTCCege egcgecg

B HNF-3 CCTAGTCTCG GTCTTGGTAG CTAACAATAT AAATGACATA CTCTGTTGTT TTCATGTITG ITITTTTTTG GGCAGACAAG GTTTCTCTCT -365

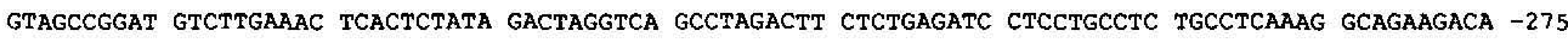

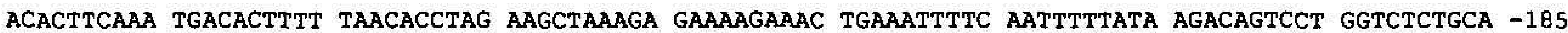
UF 1-H3 3 HNE-3 GGCAGAGAAC ACAGATCCTC CTGAAGTCAT CCCACAAGGC CCATTATTGA TTTTTTCTCC TECCCTACCC CCCACCTACT GCCCTGTTTG -95 $\mathrm{LF}-\mathrm{H} 3 \boldsymbol{\beta}$ UF $2-\mathrm{H} 3 \beta$ TITTAGTTAC GAAATGCTTT GGGCACCTTG GATTTAACTG AAAAGTAACC TTGAAACACC GAGGCCCTCA TGCCAGAGGC AAATCGCTGC -5 $+1$

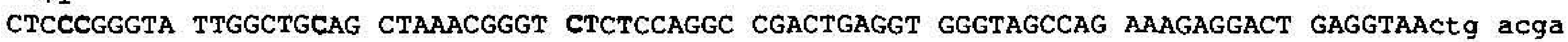

C TCTAGAGTT CCCTCCTPTA GCTGGAGATG ATGAAACACT TAGAGGCAGA AAGATTCCCC GGCTCCCGCT CTGTTGCTCT AGGGCTTTTG -230 GGATCAGCAG GGTGTGGCTC TCTACCCACG CCTTGTAGTC CCCGACTCTT CATCCATCGC AAGCTTCCAG GTGCCAGGAC CGAGGCTCCC -140 AGTGCCTGAG GTCTCTCTTC TTGCGATCCC GCAGGGGGCG CCCCAATCCG AGGGCGCCGC GCTCGGGGAG GCGCGCGGGG AGCCCGGGGC -50 TFIID $+1$ GGGCCCGGGA GGGGGTGTCC CGGCTAIAAA AAGTGGCTGC CTCCCAAGGC GCCTGGGCCA gCgggactce caggctgtgt gcctcaggt

FIG. 4. Nucleotide sequence of the $5^{\prime}$ flanking regions of the mouse HNF-3 $\alpha(\mathbf{A}), \beta(\mathbf{B})$, and $\gamma(\mathbf{C})$ genes. The transcription initiation sites determined by primer extension analysis (Fig. 3 ) are shown in boldface, and the $5^{\prime}$ most were designated as +1 . Potential binding sites for known transcription factors are underlined. The sequences present in the cDNAs are shown in lower case letters. 


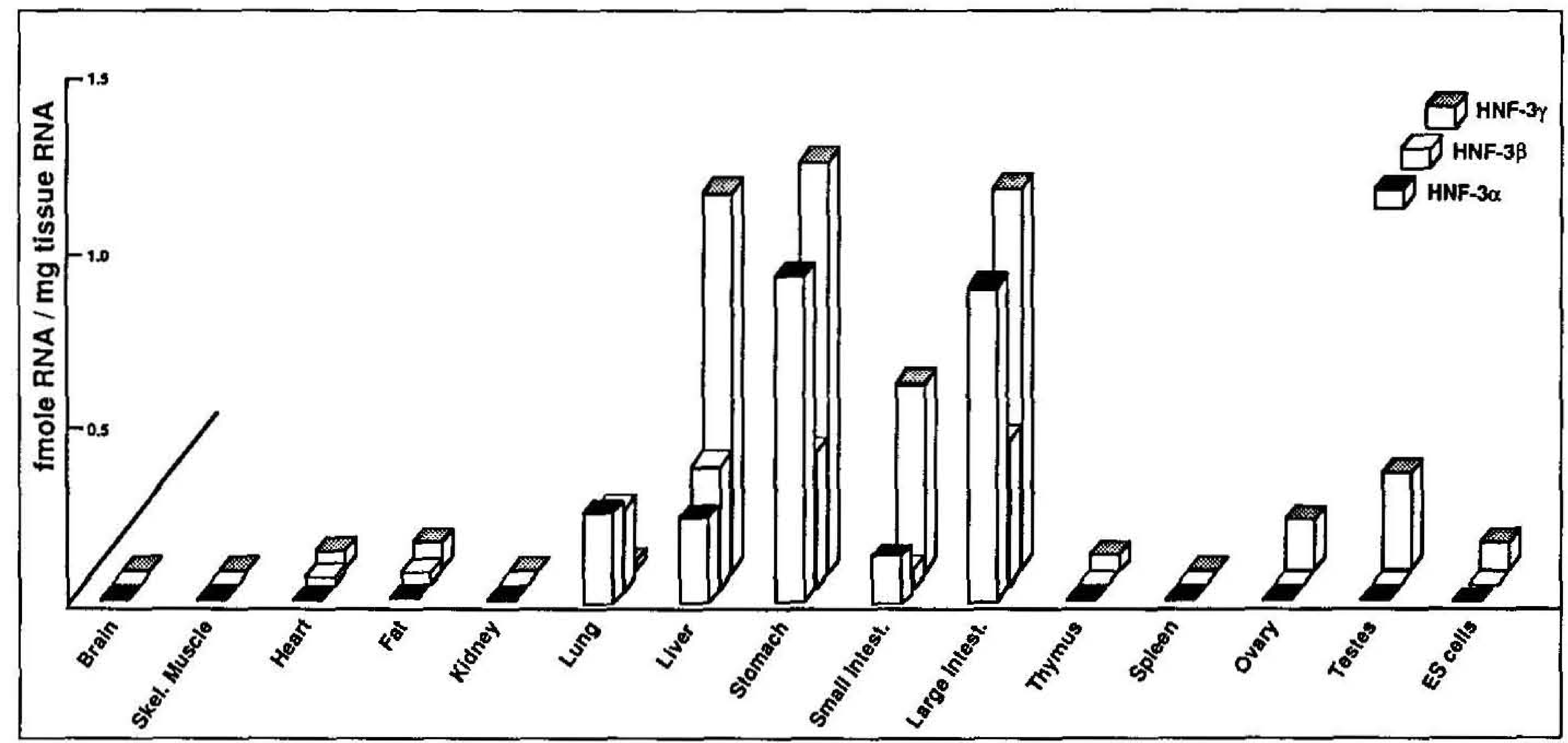

FIG. 5. Quantitative analysis of the tissue distribution of the mouse HNF- $3 \alpha, \beta$, and $\gamma$ mRNAs. (A) Fifty micrograms of total RNA from the tissues indicated was hybridized to a cocktail of excess ${ }^{32} \mathrm{P}$-labeled antisense RNA probes specific for $\mathrm{HNF}-3 \alpha$, $\beta$, and $\gamma$, and the level of the mRNAs for all three genes was determined by RNase protection analysis as described under Materials and Methods. The lane marked "Probes" contains $1000 \mathrm{dpm}$ each of the three probes used and served to standardize the values obtained in the quantitation (see below). The arrows indicate the positions of the probes $(\alpha, \beta$, and $\gamma$ ) and the protected fragments (HNF- $3 \alpha, \beta$, and $\gamma$ ). (B) The signals obtained in A were quantified using a phosphoimager and converted to fmol-specific mRNA per milligram total tissue RNA. The signals were corrected for the specific activity of the different probes and reflect the true ratios of the three mRNAs.

at $54^{\circ} \mathrm{C}$ in $80 \%$ formamide overnight. Excess probes were removed by digestion with RNases $\mathrm{A}$ and $\mathrm{T} 1$ and, the protected probe fragments analyzed on denaturing $6 \%$ polyacrylamide gels. The signals obtained were quantified on a Molecular Dynamics phosphoimager and converted to fmol RNA/mg total RNA assuming $100 \%$ hybridization of the target RNAs and normalizing the signals for the number of UMP residues incorporated in the hybridizing portion of each probe.

\section{RESULTS AND DISCUSSION}

Using a 300-nt probe spanning the forkhead DNA binding domain of the mouse HNF- $3 \alpha$ gene (Kaestner et al., 1993), we screened two mouse liver cDNA libraries. A total of 15 phages ( 10 for HNF-3 $\alpha, 1$ for HNF-3 $\beta$, and 4 
FIG. 6. Temporal expression of the HNF- $3 \alpha, \beta$, and $\gamma$ mRNAs during mouse embryogenesis. Twenty micrograms of total RNA from the developmental stages indicated (in days post coitum) were analyzed by RNase protection as described in the legend to Fig. 5. The antisense probes and protected fragments are indicated by arrows.

for $\mathrm{HNF}-3 \gamma$ ) were purified from 1.3 million phages under high-stringency conditions. The phage inserts ranged from 1 to $2.1 \mathrm{~kb}$ and contained the complete coding regions of all three genes. As demonstrated by sequence analysis and transcriptional start site mapping, the cDNA lack less than $50 \mathrm{bp}$ at the $5^{\prime}$ end. The cDNAs for HNF- $3 \beta$ and $\gamma$ are almost full length, as the corresponding mRNAs were shown to be 2.3 and $2.1 \mathrm{~kb}$ in size (data not shown). The cDNAs for HNF-3 $\alpha$ are lacking $1.6 \mathrm{~kb}$ of untranslated sequence at the $3^{\prime}$ end, as its mRNA was found to be $3.4 \mathrm{~kb}$ in length (data not shown). The sequence of the mouse HNF-3 cDNAs was determined and is shown in Fig. 1 along with the amino acid sequence for the longest open reading frame. The cDNAs encode proteins of 468 (HNF-3 $\alpha$ ), 459 (HNF$3 \beta$ ), and 353 (HNF-3 $\gamma$ ) amino acids with calculated molecular weights of $48.9,48.5$, and $34.6 \mathrm{kDa}$, respectively. As expected, sequence comparison demonstrated that the mouse and rat sequences (Lai et al., 1990, 1991) are highly conserved, with amino acid similarity ranging from $93 \%$ (for HNF-3 $\gamma$ ) to $99 \%$ (for HNF-3 $\beta$ ). A noteworthy exception is the amino terminus of the HNF-3 $\gamma$ gene, at which the mouse sequence is more similar to the mouse HNF $-3 \alpha$ and $\beta$ genes (16 of the first 55 amino acids are conserved) than to its rat counterpart ( 6 of 55 conserved amino acids). This is especially interesting in light of the finding that the amino-terminal 52 amino acids of the $\mathrm{HNF}-3 \beta$ protein were shown to be important in transcriptional activation through testing of deletion mutants in transfection assays (region IV; Pani et al., 1992a). This domain is rich in serine and tyrosine residues and contains two putative casein kinase I phosphorylation sites. Therefore, it seems likely that the amino terminus of the mouse HNF-3 $\gamma$ gene can function in transcriptional activation as well. This region of the mouse $\mathrm{HNF}-3 \gamma$ sequence was also confirmed by sequencing the corresponding genomic region (see below).

Using the mouse cDNA probes for HNF- $3 \alpha, \beta$, and $\gamma$, we screened two mouse genomic phage libraries to ob- tain genomic fragments containing all three genes. The resulting phages were mapped and the exon-containing regions identified by Southern blotting and subcloning. The restriction patterns of the cloned regions were compared to those obtained in mouse genomic Southern blots to exclude possible rearrangements of the lambda phages (data not shown). The resulting gene structures for HNF-3 $\alpha, \beta$, and $\gamma$ are shown in Fig. 2. All three genes span less than $10 \mathrm{~kb}$ and contain two (HNF-3 $\alpha$ and $\gamma$ ) or three exons (HNF-3 $\beta$ ). The intron-exon boundaries were determined by comparing the cDNA and genomic sequences and are summarized in Table 1. Interestingly, the intron within the coding region is at the same relative position in all three genes, that is, at -1 with respect to a conserved YS dipeptide, indicating a common ancestor gene. In addition, the above-mentioned divergence of the amino terminus of the mouse HNF-3 $\gamma$ gene from its rat counterpart cannot be explained by alternate exon usage, as this region covers the first 55 amino acids, while the intron is located at position 24 . In addition, no evidence for alternate splice variants of $\mathrm{HNF}-3 \gamma$ was found in RNase protection experiments using probes covering the first 452 nucleotides of the HNF-3 $\gamma$ cDNA (data not shown). The intron-exon boundaries of the rat HNF-3 $\beta$ gene, the only other HNF-3 gene analyzed so far (Pani et al., 1992b), are identical to the ones for the mouse gene described here. To determine the start site of transcription for HNF- $3 \alpha, \beta$, and $\gamma$, we performed primer extension experiments with gene-specific primers. The results of these experiments using poly $\left(\mathrm{A}^{+}\right)$RNA from stomach (where the HNF-3 gene family is expressed most strongly, see below) or tRNA as negative control are shown in Fig. 3 alongside a sequencing ladder using the same primer. In all three cases, multiple, but closely spaced, start sites are apparent. The most $5^{\prime}$ of these transcription initiation sites was designated as position +1 (see Fig. 4). The transcriptional start site of the rat HNF-3 $\beta$ gene (Pani et $a l ., 1992 b$ ) has been mapped to within $5 \mathrm{nt}$ of the one observed in mouse, again underscoring the close relationship between the two genes.

As an initial step toward the understanding of the regulatory elements governing the expression of the HNF-3 gene family, we subcloned and sequenced the proximal promoters of the three mouse genes and searched them for potential binding sites of known transcription factors (Fig. 4). Despite the relatedness of the three cDNAs and the similarity of the mRNA distribution of the three genes, the promoters are dissimilar. The promoters of $\mathrm{HNF}-3 \alpha$ and $\gamma$ have in common only binding sites for the general transcription factor Sp1 (Fig. 4), while only HNF-3 $\gamma$ contains a canonical TATA-box at the appropriate position relative to the start site of transcription. The proximal promoters of the rat and mouse HNF-3 $\beta$ genes are remarkably well conserved, with 116 of the first 120 nucleotides being identical. Especially important is the fact that the two binding sites for a liver-specific protein, termed LF-H $3 \beta$, and a binding site for HNF-3 itself are conserved in sequence as well as spac- 
ing (Fig. 4; and Pani et al., 1992b). Pani et al. (1992b) have shown through transfection of rat HNF-3 $\beta$ promoter mutants in HepG2 cells that both binding sites are important for strong expression of chimeric HNF$3 \beta /$ reporter gene constructs. They proposed a model for cell-specific transcription of the HNF-3 gene in liver cells involving activation by the cell-specific LF-H3 $\beta$ protein and maintenance of this expression through positive autoregulation by HNF-3 $\beta$. Interestingly, a second HNF-3 binding site of identical sequence is found farther upstream (position -400 to -390 ) in the mouse HNF-3 $\beta$ promoter, which might also take part in this proposed autoregulation. A second possibility is the regulation of the HNF- $3 \beta$ promoter by $\mathrm{HNF}-3 \alpha$ and $\gamma$, which are also present in liver and have similar DNA binding properties (Lai et al., 1991). Most likely, similar regulatory pathways are operative in the regulation of the mouse and rat HNF- $3 \beta$ genes. This notion is supported by the finding that the proximal mouse HNF-3 $\beta$ promoter confers cell-type-specific expression on a $\beta$-galactosidase reporter gene (data not shown). A detailed promoter analysis of the divergent HNF- $3 \alpha$ and $\gamma$ genes through transfection as well as transgenic mouse experiments is in progress to understand which regulatory pathways control these genes, as their proximal promoters lack binding sites for HNF-3 and LF-H3 $\beta$.

As a first step toward understanding the full potential of the HNF-3 gene family as transcription factors in mice, we studied the expression patterns of its three members through quantitative RNase protection using gene-specific probes (see Materials and Methods for details), allowing for a first direct comparison of the expression levels of the three genes. The results of our survey of adult mouse tissues are shown in Fig. 5. While HNF- $3 \alpha$ is expressed strongly in large intestine and stomach, and weaker in liver, lung, and small intestine, HNF- $3 \beta$ mRNA is present in the same tissues at more balanced levels. HNF-3 $\gamma$ is expressed strongly in large and small intestine, stomach, and liver, but is absent from lung. This is consistent with the observation of Monaghan et al. (1993) that HNF-3 $\gamma$ expression in midgestation mouse embryos is restricted to derivatives of the endoderm posterior to the liver. The expression of HNF $-3 \alpha$ and $\beta$ in the adult mouse is restricted to derivatives of the embryonic endoderm, while HNF- $3 \gamma$ mRNA was also found in heart, adipose tissue, ovary, and testis as well as embryonic stem cells. The tissue distribution of the HNF-3 mRNAs in mice is qualitatively similar to that observed in the rat (Sladek and Darnell, 1992; Lai et $a l ., 1991)$. The expression of $\mathrm{HNF}-3 \gamma$ in heart, adipose tissue and embryonic stem cells has not been observed before. We extended our analysis of the expression of the HNF-3 gene family to RNAs obtained from mouse embryos from Day 9.5 post coitum onward using the same gene-specific probes (Fig. 6). HNF-3 $\beta$ is expressed strongly on Days 9.5 and 10.5, weakly detected during the next 2 days, and returns to an intermediate level of expression on Day 15.5. HNF-3 $\alpha$ follows a similar pattern as HNF- $3 \beta$, but is expressed at lower levels. The transient dip in the mRNA levels of HNF-3 $\alpha$ and $\beta$ corresponds to the disappearance of the two mRNAs from the embryonic liver as revealed by in situ hybridization (Monaghan et al., 1993). HNF-3 $\gamma$, while hardly detectable on Day 9.5, is being activated on Day 12.5 and peaks on Days 15.5 and 16.5. This peak in mRNA levels coincides with the increased expression of HNF- $3 \gamma$ in endoderm-derived structures such as the liver, stomach, intestine, and pancreas observed by in situ hybridization (Monaghan et al., 1993).

As an initial step toward understanding the role of the HNF-3 gene family of transcription factors, we have cloned and characterized the cDNAs and genes of its three members. The information obtained will allow identification of important regulatory elements in the promoters of HNF- $3 \alpha, \beta$, and $\gamma$ through transfection of chimeric promoter/reporter gene constructs into tissue culture cells and by analysis of these constructs in transgenic mice. Furthermore, gene targeting experiments in mouse embryonic stem cells that will allow us to determine the precise role of these genes in mouse embryonic development and tissue-specific transcriptional regulation in the adult are in progress.

\section{ACKNOWLEDGMENTS}

We thank Drs. A. P. Monaghan and J. Blendy for critically reading the manuscript, B. Lupp and $H$. Kern for technical assistance, and W. Fleischer for oligonucleotide synthesis as well as photographic work. This work was supported by the Deutsche Forschungsgemeinschaft through Sonderforschungsbereich 229, the Leibniz-Programm, and Fonds der Chemischen Industrie.

\section{REFERENCES}

Auge-Gouillou, C., Petropoulos, I., and Zakin, M. M. (1993). Liverenriched HNF-3 alpha and ubiquitous factors interact with the human transferrin gene enhancer. FEBS Lett. 323: 4-10.

Chirgwin, J. M., Przybyla, A. E., MacDonald, R. J., and Rutter, W. J. (1979). Isolation of biologically active ribonucleic acid from sources enriched in ribonuclease. Biochemistry 18: 5294-5299.

Church, G. M., and Gilbert, W. (1984). Genomic sequencing. Proc. Natl. Acad. Sci. USA 81: 1991-1995.

Clevidence, D. E., Overdier, D. G., Tao, W., Qian, X., Pani, L., Lai, E., and Costa, R. H. (1993). Identification of nine tissue-specific transcription factors of the HNF-3/forkhead DNA binding domain family. Proc. Natl. Acad. Sci. USA 90: 3948-3952.

Costa, R. H., Grayson, D. R., and Darnell, J. E. (1989). Multiple hepa. tocyte-enriched nuclear factors function in the regulation of transthyretin and $\alpha-1$ antitrypsin genes. Mol. Cell. Biol. 9: 14151425.

De Simone, V., and Cortese, R. (1991). Transcriptional regulation of liver-specific gene expression. Curr. Opin. Cell Biol. 3: 960-965.

Dirksen, M. L., and Jamrich, M. (1992). A novel, activin-inducible, blastopore lip-specific gene of Xenopus laevis contains a forkhead DNA binding domain. Genes Dev. 6: 599-608.

Frischauf, A. M. (1987). Construction and characterization of a genomic library in $\lambda$. Methods Enzymol. 152: 190-199.

Häcker, U., Grossniklaus, U., Gehring, W. J., and Jäckle, H. (1992). Developmentally regulated Drosophila gene family encoding the fork head domain. Proc. Natl. Acad. Sci. USA 89: 8754-8758.

Henikoff, S. (1984). Unidirectional digestion with exonuclease III 
creates targeted breakpoints for DNA sequencing. Gene 28: $351-$ 355.

Herbst, R. S., Nielsch, U., Sladek, F., Lai, E., Babiss, L. E., and Darnell, J. E. (1991). Differential regulation of hepatocyte-enriched transcription factors explains changes in albumin and transthyretin gene expression among hepatoma cells. New Biol. 3: 289-296.

Hooper, M., Hardy, K., Handyside, A., Hunter, S., and Monk, M. (1987). HPRT-deficient (Lesch-Nyhan) mouse embryos derived from germline colonization by cultured cells. Nature 326: 292-295.

Ip, Y. T., Poon, D., Stone, D., Granner, D. K., and Chalkley, R. (1990). Interaction of a liver-specific factor with an enhancer 4.8 kilobases upstream of the phosphoenolpyruvate carboxykinase gene. $\mathrm{Mol}$. Cell. Biol. 10: $3770-3781$.

Jürgens, G., and Weigel, D. (1988). Terminal versus segmental development in the Drosophila embryo: The role of the homeotic gene forkhead. Roux's Arch. Dev. Biol. 197: 345-354.

Kaestner, K. H., Lee, K.-H., Schlöndorff, J., Hiemisch, H., Monaghan, A. P., and Schütz, G. (1993). Six members of the mouse forkhead gene family are developmentally regulated. Proc. Natl. Acad. Sci. USA 90: 7628-7631.

Kaestner, K. H., Ntambi, J. M., Kelly, T. J., and Lane, M. D. (1989) Differentiation-induced gene expression in 3T3-L1 preadipocytes: A second differentially expressed gene encoding stearoyl-CoA desaturase. J. Biol. Chem. 264: 14755-14761.

Knöchel, S., Lef, J., Clement, J., Klocke, B., Hille, S., Köster, M., and Knöchel, W. (1992). Activin A induced expression of a fork head related gene in posterior chordamesoderm (notochord) of Xenopus laevis embryos. Mech. Dev. 38: 157-165.

Kuo, C. J., Conley, P. B., Chen, L., Sladek, F. M., Darnell, J. E., and Crabtree, G. R. (1992). A transcriptional hierarchy involved in mammalian cell-type specification. Nature $355 ; 458-460$.

Lai, E., and Darnell, J. E. (1991). Transcriptional control in hepatocytes: A window on development. Trends Biochem. Sci. 16: 427430.

Lai, E., Prezioso, V. R., Smith, E., Litvin, O., Costa, H. R., and Darnell, J. E., Jr. (1990). HNF3A, a hepatocyte-enriched transcription factor of novel structure is regulated transcriptionally. Genes Dev. 4: $1427-1436$.

Lai, E., Prezioso, V. R., Tao, W., Chen, W. S., and Darnell, J. E., Jr. (1991). Hepatocyte nuclear factor $3 \alpha$ belongs to a family in mammals that is homologous to the Drosophila homeotic gene forkhead. Genes Dev. 5: 416-427.

Li, C., Lai, C., Sigman, D. S., and Gaynor, R. B. (1991). Cloning of a cellular factor, interleukin binding factor, that binds to NFAT-like motifs in the human immunodeficiency virus long terminal repeats. Proc. Natl. Acad. Sci. USA 88: 7739-7743.

Li, C., Lusis, A. J., Sparkes, R., Tran, S.-M., and Gaynor, R. (1992). Characterization and chromosomal mapping of the gene encoding the cellular DNA binding protein HTLF. Genomics 13: 658-664.

$\mathrm{Li}$, J., and Vogt, P. K. (1993). The retroviral oncogene qin belongs to the transcription factor family that includes the homeotic gene fork head. Proc. Natl. Acad. Sci. USA 90: 4490-4494.

Luckow, B., Bunz, F., Stillman, B., Lichter, P., and Schütz, G. (1994).
Cloning, expression and chromosomal localization of the $140 \mathrm{kDa}$ subunit of replication factor $\mathrm{C}$ from mouse and man. Mol. Cell. Biol., in press.

McPherson, C. E., Shim, E.-Y., Friedman, D. S., and Zaret, K. S. (1993). An active tissue-specific enhancer and bound transcription factors existing in a precisely positioned nucleosomal array. Cell 75: $387-398$.

Monaghan, A. P., Kaestner, K. H., Grau, E., and Schütz, G. (1993). Post-implantation expression patterns indicate a role for the mouse forkhead/HNF-3 $\alpha, \beta$ and $\gamma$ genes in determination of the definite endoderm, chordamesoderm and neuroectoderm. Development 119: 567-578.

Nitsch, D., Boshart, M., and Schütz, G. (1993). Activation of the tyrosine aminotransferase gene is dependent on synergy between liverspecific and hormone-responsive elements. Proc. Natl. Acad. Sci. USA 90: $5479-5483$.

Oliver S. G., et al. (1992). The complete DNA sequence of yeast chromosome III. Nature 357: 38-46.

Pani, L., Overdier, D. G., Porcella, A., Qian, X., Lai, E., and Costa, R. H. (1992a). Hepatocyte nuclear factor $3 \beta$ contains two transcriptional activation domains, one of which is novel and conserved with the Drosophila fork head protein. Mol. Cell. Biol. 12: 3723-3732.

Pani, L., Qian, X., Clevidence, D., and Costa, R. H. (1992b). The restricted promoter activity of the liver transcription factor hepatocyte nuclear factor $3 \beta$ involves a cell-specific factor and positive autoregulation. Mol. Cell. Biol. 12: 552-562.

Ruiz i Altaba, A., and Jessell, T. M. (1992). Pintallavis, a gene expressed in the organizer and midline cells of frog embryos: Involvement in the development of the neural axis. Development 116: 8193.

Ruppert, S., Boshart, M., Bosch, F. X., Schmid, W., Fournier, R. E. K., and Schütz, G. (1990). Two genetically defined trans-acting loci coordinately regulate overlapping sets of liver-specific genes. Cell 61: 895-904.

Sanger, F., Nicklen, S., and Coulson, A. R. (1977). DNA sequencing with chain-terminating inhibitors. Proc. Natl. Acad. Sci. USA 74: 5463-5467.

Sasaki, H., and Hogan, B. L. M. (1993). Differential expression of multiple fork head related genes during gastrulation and axial pattern formation in the mouse embryo. Development 118: 47-59.

Sawaya, P. L., Stripp, B. R., Whitsett, J. A., and Luse, D. S. (1993). The lung-specific $\mathrm{CC} 10$ gene is regulated by transcription factors from the AP-1, octamer, and hepatocyte nuclear factor 3 families. Mol. Cell. Biol. 13: 3860-3871.

Sladek, F. M., and Darnell, J. E. (1992). Mechanisms of liver-specific gene expression. Curr. Opin. Genet. Dev. 2: 256-259.

Tao, W., and Lai, E. (1992). Telencephalon-restricted expression of BF-1, a new member of the HNF-3/fork head gene family in the developing rat brain. Neuron 8: 957-966.

Weigel, D., and Jäckle, H. (1990). The fork head domain: A novel DNA binding motif of eucariotic transcription factors. Cell 63: 455-456.

Weigel, D., Jürgens, G., Küttner, F., Seifert, E., and Jäckle, H. (1989). The homeotic gene fork head encodes a nuclear protein and is expressed in the terminal regions of the drosophila embryo. Cell 57: 645-658. 